\title{
Intracoronary adenosine-induced torsades de pointes during fractional flow reserve measurement
}

\author{
Sophie Degrauwe ${ }^{1}$, Alessia Azzano ${ }^{2}$, Juan F. Iglesias ${ }^{1}$, Carlo Zivelonghi², \\ Stefan Verheye ${ }^{2}$, Carl Convens ${ }^{2}$, Paul Vermeersch ${ }^{2}$, Benjamin Scott ${ }^{2}$ \\ ${ }^{1}$ Department of Cardiology, Geneva University Hospital, Geneva, Switzerland \\ ${ }^{2}$ Department of Cardiology, ZNA Middelheim, Antwerp, Belgium
}

We report a 58-year-old woman with multiple cardiovascular risk factors presenting with stable angina. Echocardiography demonstrated normal systolic left ventricular function (ejection fraction 65\%), with the absence of structural abnormalities. The patient was referred for coronary angiography, demonstrating angiographic intermediate stenosis in the proximal segment of the intermediate artery (IA) and left anterior descending artery (LAD) (Fig. 1).
Fractional flow reserve (FFR) measurement using adenosine $(240 \mu \mathrm{g}$, volume $20 \mathrm{~mL})$ was performed in the IA as well as LAD, both measures were negative, respectively 0.97 and 0.84 (Fig. 2). Following immediate intracoronary (IC) adenosine administration in the LAD the patient developed torsades de pointes (TdP) with circulatory collapse (Fig. 2). Intra-procedural electrocardiogram revealed supraventricular extra-systoles with short coupling

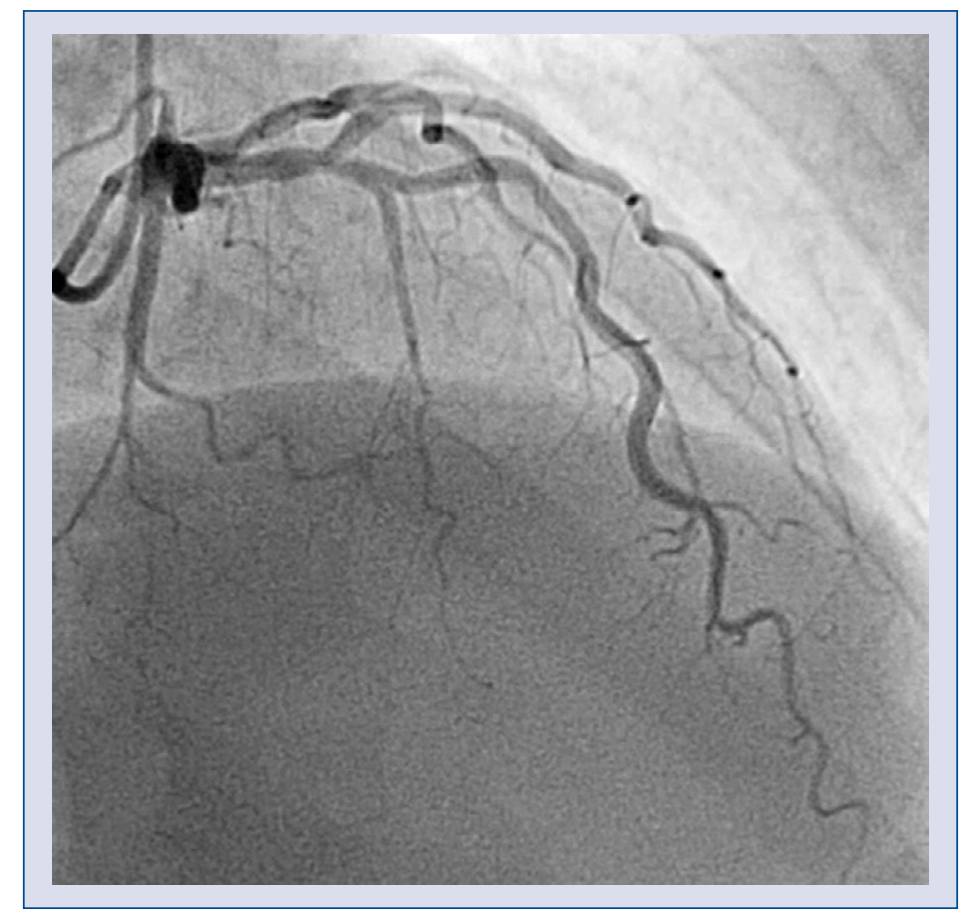

Figure 1. Coronary angiogram demonstrating angiographic intermediate stenosis of the proximal left anterior descending artery.

Address for correspondence: Sophie Degrauwe, MD, Department of Cardiology, Hôpitaux Universitaires Genève,

Rue Gabrielle-Perret-Gentil 4, 1205 Genève, Switzerland, tel: +41 7955302 94, fax: +41 2237272 29,

e-mail: sophie.degrauwe@hcuge.ch

Received: 13.05.20199 Accepted: 19.08.2019 


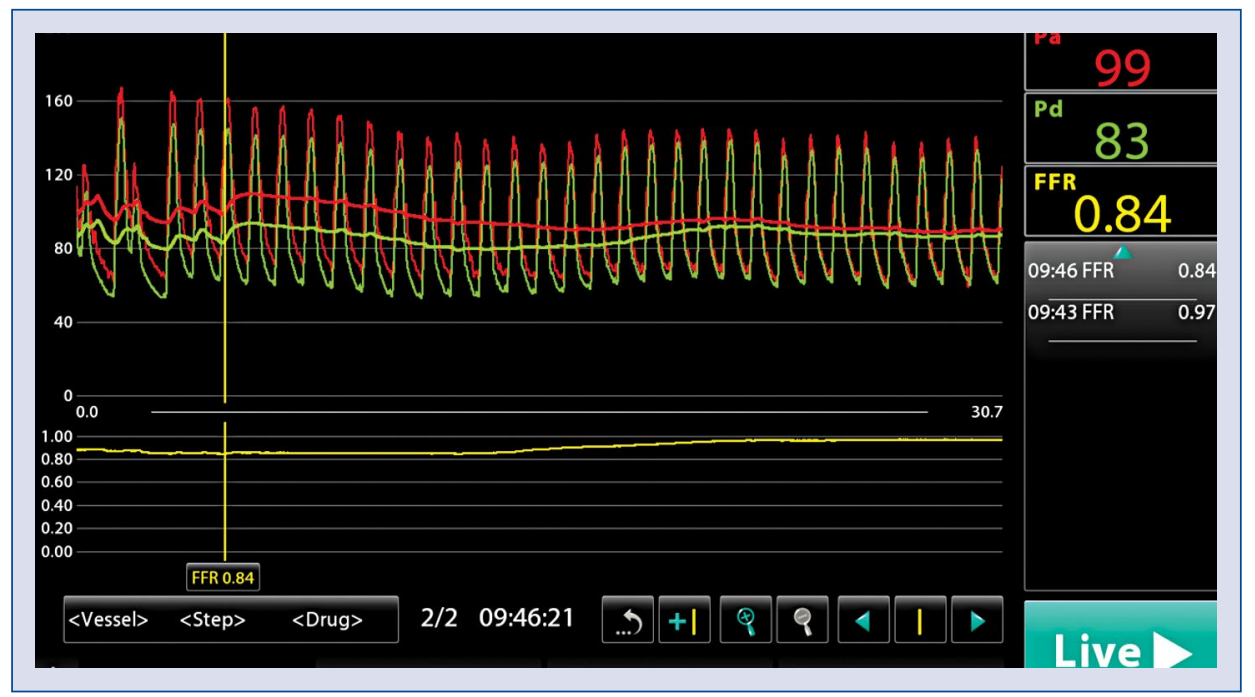

Figure 2. Fractional flow reserve (FFR) console screenshot demonstrating FFR values measured in the intermediate artery (0.97) and left anterior descending artery (0.84).

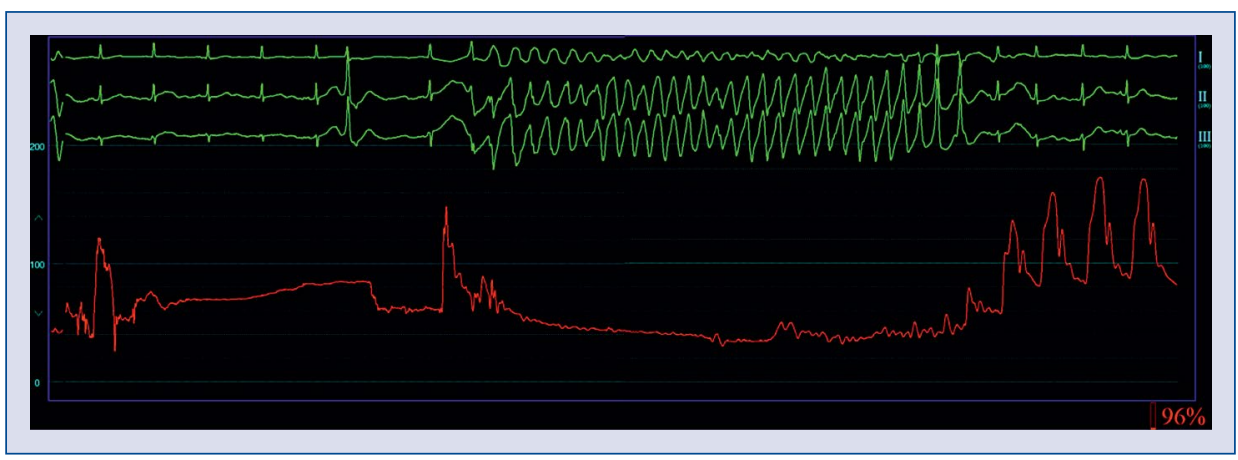

Figure 3. Electrocardiogram and arterial pressure following immediate intra-coronary adenosine administration for fractional flow reserve measurement of the left anterior descending artery, leading to torsades de pointes, with spontaneous self-termination.

at the time of adenosine injection, preceding the onset of TdP (Fig. 3).

Recognized adenosine-induced ventricular arrhythmias (VA) include (1) ventricular fibrillation (VF) in pre-excited atrial fibrillation, (2) polymorphic ventricular tachycardia (VT) in long Q-T syndromes, (3) degeneration of VT to VF and (4) non-sustained VT following termination of supraventricular tachycardia. VA induced by adenosine for FFR assessment is a rare complication described in 5 cases in available literature, occurring after adenosine administration in patients with FFR positive and negative lesions, suggesting a lack of causality between myocardial ischemia and the onset of VA. The mechanisms of VA induced by IC adenosine administration might be related to adenosine dose and concentration, saline bolus injection volume, or pharmacological effect of adenosine (induces VF by "R on T" phenomenon with or without atrioventricular block). This is the first description of IC adenosine induced TdP with circulatory collapse. The mechanism seems to be at least partially linked to adenosine induced enhanced ventricular automaticity. FFR is currently considered the gold standard for the functional assessment of coronary stenosis; however, its measurement warrants the need of adenosine. The present case highlights that caution is of the essence when performing adenosine injection for an FFR measurement in a patient presenting extrasystoles with short coupling. 